
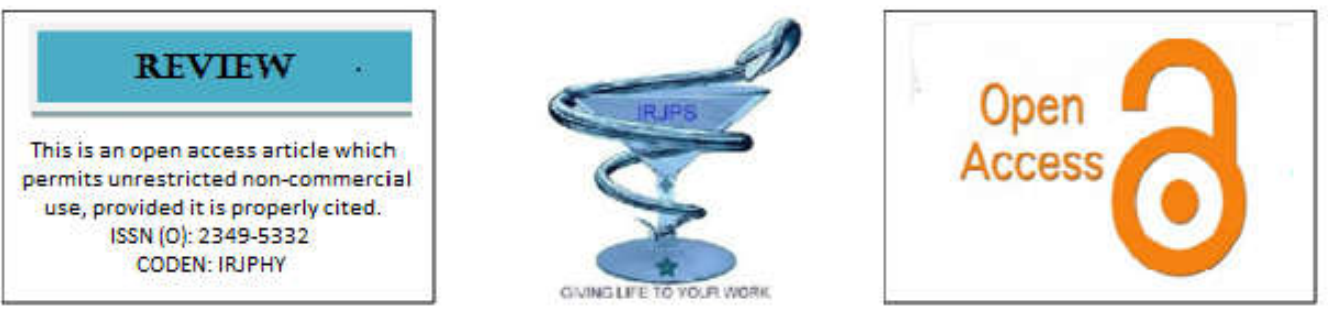

\title{
DRUG - DRUG INTERACTION: AN IMPORTANT DRUG RELATED PROBLEM
}

\author{
Swathi V $S^{1 *}$, Joshna Rani $S^{2}$ \\ ${ }^{1}$ Vignan Institute of Pharmaceutical Technology, Duvvada, Visakhapatnam \\ ${ }^{2}$ Institute of Pharmaceutical Technology, Sri Padmavathi Mahila Viswavidyalayam, Tirupati, Andhra \\ Pradesh
}

Submitted on: 30.09.19;

Revised on: 06.11.19;

Accepted on: 11.11.19

\begin{abstract}
Drug interaction is defined as the clinical or pharmacological response occurs when two drugs administered simultaneously, results in beneficial or harmful effects in a person. Based upon mechanism of action drug interactions are classified in to three types: Pharmacokinetic interactions, Pharmacodynamic interactions, and Pharmaceutical interactions. Based upon severity, drug interactions are classified in to four types: Minor, moderate, major and contraindicated. According to WHO drug interactions are main cause of mortality and morbidity. It has been estimated that prevalence of interactions is estimated to be 1$22 \%$ in world. Drug- drug interactions accounted for $1.1 \%$ hospital admissions and $0.1 \%$ hospital visits. Polypharmacy, Age, Prescribing errors, some departments and some disease conditions are the reasons for drug-drug interactions. These interactions lead to misinterpretation, alteration of affinity and efficacy of drugs, increased hospital cost, stay and admission, increased morbidity and mortality and Increased incidence of adverse events and adverse drug reactions. Clinical pharmacist assisted computerised decision support systems will reduce alert fatigue by pharmacist decision on drug - drug interactions to avoid drug related problems. As these drug interactions effects the safety of patient health care professions should focus on these in clinical settings.
\end{abstract}

Keywords: Drug interactions, Morbidity, Mortality, Adverse effects, Polypharmacy

Corresponding author: V.S. Swathi
E-mail: swathipharmacoo@gmail.com $\begin{gathered}\text { Indian Research Journal of Pharmacy and Science; 22(2019)2008-2012; } \\ \text { Journal Home Page: https://www.irjps.in } \\ \text { DOI: 10.21276/irjps.2019.6.3.11 }\end{gathered}$




\section{INTRODUCTION:}

\section{What is Drug interaction?}

Drug interaction is defined as the clinical or pharmacological response occurs when two drugs administered simultaneously, results in beneficial or harmful effects in a person. ${ }^{1}$

There are both beneficial and harmful effects with combination of two or more drugs. Some studies mentioned that, there is positive correlation between number of drugs consumed and harmful effects observed. ${ }^{2}$

\section{What mechanisms involved in Drug interactions?}

Based upon mechanism of action drug interactions are classified in to three types. They are Pharmacokinetic interactions, Pharmacodynamic interactions, and Pharmaceutical interactions.

Pharmacokinetic interactions, occurs when two drugs administered simultaneously, one drug may lead to changes in absorption, distribution, metabolism and excretion of other drug results in decreased or increased effect of other drug.

Pharmacodynamic interactions, occurs when two drugs administered simultaneously, one drug may lead to changes in affinity and efficacy of other drug results in synergistic or additive or antagonistic effect.

Pharmaceutical interactions, occurs when two chemically incompatible drugs co administered, leading to precipitation and inactivation of the therapeutic effects of the drugs ${ }^{3}$

Based upon severity, drug interactions are classified in to four types: Minor, moderate, major and contraindicated. No interventions required in case of minor interactions. Monitoring of drug therapy is required in case of moderate interactions. Severe adverse outcomes can occur in case of major interactions, so interventions like dose, frequency, route modification or selection of alternative drugs may be needed. In case of contraindication, drug combination must be avoided. ${ }^{4}$

According to $\mathrm{WHO}$ and previous published literature, drug interactions are main cause of mortality and morbidity in susceptible population (in elders where polypharmacy is possible). So minimising and prevention of these interactions is required to improve quality of care in both outpatient and inpatient settings. ${ }^{5}$

\section{Epidemiology of Drug interactions}

It has been estimated that prevalence of interactions is estimated to be $1-22 \%$ in world. Drug- drug interactions accounted for $1.1 \%$ hospital admissions and $0.1 \%$ hospital visits. Some studies mentioned that drug- drug interactions are responsible for $20-30 \%$ of all drug side effects, of which $70 \%$ need clinical attention and 1-2\% cases lead to life-threatening situations. Total $25-47 \%$ of clinically significant drug- drug interactions were common in elders. According to some studies $60 \%$ of inpatients in general medical wards and $16-47 \%$ of patients in emergency departments were at risk of clinically relevant drug interactions. Prevalence of major drug interactions were $63.5 \%$ and moderate drug interactions were $60.5 \%{ }^{6}$

Why these drug interactions occurs?

Drug - drug interactions occurs due to following reasons:

\section{Polypharmacy}

It is a condition where patient take many drugs at the same time. This results in drug - drug interactions. Many studies proved that this is a reason for clinically significant reactions. There is a direct relationship between number of drugs taken and occurrence of drug interactions. If the person consumes more than 5 drugs, there will be above $50 \%$ chance of incidence of drug- drug interactions. $^{7}$

\section{Age}

Due to altered pharmacokinetics and pharmacodynamics variations in elders drug interactions can occur easily. Co morbid conditions are more common in elders, such that they have to take multiple medications for treating different conditions which leads to increased incidence of drug- drug interactions. ${ }^{8}$

\section{Prescribing errors}

Prescribing multiple medications without checking risk of interactions is another reason for drug- drug interactions . 


\section{Departments}

Especially few departments have high risk of getting DDIs. For example cardiology department, inpatient wards, emergency departments and geriatric ward where prescribing of drugs is a complex process. ${ }^{10}$

\section{Disease conditions}

Cancer patients using chemotherapy drugs and HIV patients using antiretroviral drugs will have more chance of getting drug - drug interaction related adverse effects. ${ }^{11}$

\section{What are the consequences of drug interactions?}

\section{Misinterpretation}

Effects of drug-drug interactions lead to misjudgement and misinterpretation as new disease. $^{12}$

\section{Alteration of safety and efficacy}

DDIs may alter efficacy and safety of other drug results in alteration of therapeutic response or increase in adverse effects of drugs. ${ }^{13}$

3. Increased hospital admission, stay and cost

These leads to hospital admissions, increased length of hospital stay and increases treatment cost on patient. ${ }^{13}$

4. Increased morbidity and mortality

Some drug interactions are responsible for mortality and morbidity. ${ }^{14}$

5. Increased incidence of adverse events and adverse drug reactions

Many DDIs are responsible for ADRs and ADRs like hepatotoxicity, nephrotoxicity and anemia etc. ${ }^{15}$

\section{How will you prevent these drug interactions?}

By framing and implementation of guidelines for the identification of drug interactions can help the physicians and pharmacists to avoid drug drug interaction related problem. ${ }^{16}$
Clinical case discussions related to major interactions would helpful in framing of protocols. $^{17}$

Clinical pharmacists play important role in identification, monitoring and management of drug- drug interactions in both inpatient and outpatient settings which is a main role to improve patient safety and quality of care in hospital. Peer review of prescriptions before dispensing is a good method to prevent drugdrug interactions. Clinical pharmacist assisted computerised decision support systems will reduce alert fatigue by pharmacist decision on drug - drug interactions to avoid drug related problems. $^{18}$

There are some softwares with high specificity and reliability to identify drug interactions. For example, Micromedex and Lexi-interact. ${ }^{19}$

By establishing having drug information centers in hospitals drug drug interaction related information can be provided easily to all health care professions and to patients to increase awareness about drug safety information. ${ }^{20}$

Computerized decision support system connected with electronic medical records in another way of identification and management of drug - drug interactions. But main disadvantage is expensive installation and alert fatigue leads to under use of this systems. ${ }^{21}$

Documentation of drug- drug interactions is very important area to share knowledge about drugdrug interactions in a clinical setting and multidisciplinary approaches and improved communication among health care professionals also very important to improve patient safety. ${ }^{22}$

Some previously clinically observed adverse drug reactions due to drug-drug interactions. ${ }^{23}$

Those are as follows:

- Rifampicin and Pyrazinamide

- Rifampicin and Isoniazid

- Ciprofloxacin and Theophylline

- Rifampicin and Nevirapine

- Warfarin and Paclitaxel

- Warfarin and Ifosfamide

- Warfarin and Etoposide

- Warfarin and Carboplatin 
- Warfarin and Trimethoprim / Sulfamethoxazole

- Warfarin and Metronidazole

- Warfarin and Fluconazole

- Warfarin and Ciprofloxacin

- Warfarin and Levofloxacin

- Warfarin and Clarithromycin

- Warfarin and Statin

- Omeprazole and clopidogrel

- Clarithromycin and Simvastatin

- Clarithromycin and Ciprofloxacin

- Low-dose aspirin and Clopidogrel

- Angiotensin-converting-enzyme inhibitor and Aldosterone

- ACE inhibitor and Potassium sparing diuretic

- B-adrenergic antagonist and B-2 agonist

- NSAIDs and Anti hypertension therapy

- Digoxin and Calcium channel blockers

- Digoxin and Beta-blockers

There are some limitations involved in previous studies related to drug interactions. ${ }^{24}$

Those are:

- Short period of investigation

- No interventions regarding the drug interactions identified were suggested/ performed

\section{REFERENCES:}

1. Brunton LL, et al. Goodman \& Gilman: As Bases Farmacológicas da Terapêutica. 11th ed. Rio de Janeiro: McGraw-Hill Interamericana do Brasil. 2006.

2. Hartshorn EA. Drug interaction: 1. General considerations. Ann Pharmacother. 2006; 40: 116-8.

3. Blower P, de Wit R, Goodin S, Aapro M. Drug-drug interactions in oncology: why are they important and can they be minimized? Crit Rev Oncol Hematol. 2005; 55: 117-42.

4. Armahizer MJ, et al. Comparing drugdrug interaction severity ratings between bedside clinicians and proprietary databases. ISRN Crit Care. 2013; 2013:6
- Patient cases were not entirely considered, as only prescriptions were verified

- Effect of drug interactions on patient outcomes was not considered

- Difficulty in detecting clinically significant reactions

- Only well supported interactions with literature are included in all studies

The best strategy to prevent DDIs is still unknown. ${ }^{25}$

\section{CONCLUSION}

In most of the clinical settings considering about drug- drug interactions are neglected due to lack of awareness, complex treatment, lack of time and lack of manpower. As they are having high significance on safety of the patient focus must be kept to minimise drug-drug interaction induced adverse effects. Prospective observational studies should be carried out to know about clinically significant drug-drug interactions. Drug-drug interaction softwares like Micromedex and Lexicomp should be used to identify drug interactions. Clinical pharmacists should take active role in identification, monitoring and management of drug interactions.

5. Riechelmann RP, Del Giglio A. Drug interactions in oncology: how common are they? Ann Oncol 2009:1907-1912

6. Dechanont $\mathrm{S}$, et al. Hospital admissions/ visits associated with drug-drug interactions: a systematic review and meta-analysis. Pharmacoepidemiol Drug Saf. 2014; 23: 489-97.

7. Hussar DA. Drug interaction. In: GennaroL, Marderosian A, Hanson G et al. Remington The science and practice of pharmacy. Philadelphia: seventh edition: Lippincott Williams and Willinks 2007. 1746-61.

8. Marzolini $\mathrm{C}$, et al. Ageing with HIV: medication use and risk for potential drug-drug interactions. J Antimicrob Chemother. 2011;66(9):2107-1. 
9. Foroughinia F, Tazarehie SR, Petramfar P. Detecting and managing drug-related problems in the neurology ward of a tertiary care teaching hospital in Iran: a clinical pharmacist's intervention. J Res Pharm Pract. 2016; 5(4):285-9.

10. Lenssen $\mathrm{R}$, et al. Analysis of drugrelated problems in three departments of a German University hospital. Int $\mathbf{J}$ Clin Pharm. 2016; 38(1):119-26.

11. Patel VK, et al. Potential drug interactions in patients admitted to cardiology wards of a south Indian teaching hospital. Australas Med J 2011; 4: 9-14.

12. Seymour R, Routledge P. Important drug-drug interactions in the elderly. Drugs Aging. 1998; 12(6):485-94.

13. Patel VK, et al. Potential drug interactions in patients admitted to cardiology wards of a south Indian teaching hospital. Australas Med J. 2011; 4:9-14

14. Buajordet I, et al. Fatal adverse drug events: The paradox of drug treatment. J Intern Med 2001; 250:327-41.

15. Pirmohamed $M$, et al. Adverse drug reactions as cause of admission to hospital: prospective analysis of 18,820 patients. BMJ. 2004; 329: 1519.

16. Moura CS, Acurcio FA, Belo N. Drugdrug interactions associated with length of stay and cost of hospitalization. J Pharm Pharm Sci. 2009; 12(3): 266-72.

17. Patricia Moriel et al. Drug interactions in female oncologic inpatients: differences among databases. Rev
Ciênc Farm Básica Apl., 2013;34(3):333-337.

18. Dean B, et al. Prescribing errors in hospital inpatients: their incidence and clinical significance. Qual Saf Health Care. 2002; 11(4):340-4.

19. Barrons R. Evaluation of personal digital assistant software for drug interactions. Am J Health Syst Pharm. 2004; 61(4):380-5.

20. Entezari-Maleki T, et al. A two-year utilization of the pharmacist-operated drug information center in Iran. J Res Pharm Pract 2014; 3:117-22.

21. Schnipper JL, et al. Effect of an electronic medication reconciliation application and process redesign on potential adverse drug events: a cluster-randomized trial. Arch Intern Med. 2009; 169(8):771-80.

22. Norton PG, Baker GR. Patient safety in cancer care: a time for action. J Natl Cancer Inst. 2007; 99:579-80.

23. Cooney D, Pascuzzi K. Polypharmacy in the elderly: focus on drug interactions and adherence in hypertension. Clin Geriatr Med. 2009; 25(2):221-33.

24. Joseph V et al, The Prevalence of Opioid-Related Major Potential DrugDrug Interactions and Their Impact on Health Care Costs in Chronic Pain Patients, Journal of Managed Care \& Specialty Pharmacy JMCP.2014; 20(5):467-476.

25. Riechelmann RP. Drug combinations with the potential to interact among cancer patients. Support Care Cancer. 2007;15:1113-4. 